\title{
A Note on Pseudo-Umbilical Submanifolds of Hessian Manifolds with Constant Hessian Sectional Curvature
}

\author{
Münevver Yildirim Yilmaz and Mehmet Bektaş \\ Department of Mathematics, Faculty of Arts and Science, Firat University, 23119 Elazig, Turkey \\ Correspondence should be addressed to Münevver Yildirim Yilmaz, munyildirim@gmail.com
}

Received 22 March 2011; Accepted 26 April 2011

Academic Editor: S. Sivasubramanian

Copyright (C) 2011 M. Yildirim Yilmaz and M. Bektaş. This is an open access article distributed under the Creative Commons Attribution License, which permits unrestricted use, distribution, and reproduction in any medium, provided the original work is properly cited.

The geometry of Hessian manifold, as a branch of statistics, physics, Kaehlerian, and affine differential geometry, is deeply fruitful and a new field for scientists. However, inspite of its importance submanifolds and curvature conditions of it have not been so well known yet. In this paper, we focus on the pseudo-umbilical submanifolds on Hessian manifold with constant Hessian sectional curvature and using sectional curvature conditions we obtain new results on it.

\section{Introduction}

A Riemannian metric on a flat manifold is called a Hessian metric if it is locally expressed by the Hessian of functions with respect to affine coordinate systems. The pair of $(D, g)$ with flat connection $D$ and Hessian metric $g$ is called Hessian structure, and a manifold equipped with this structure is said to be a Hessian manifold. In [1, 2], Hirohiko Shima introduced Hessian sectional curvature and its relations with Kaehlerian manifold. He also proved theorems and gave important remarks on the spaceform of Hessian manifolds. In the light of these studies Bektaş et al. obtained some curvature conditions, results, and integral inequalities on this type of manifolds, [3-5].

Let $M^{n+p}$ be an $(n+p)$-dimensional Hessian manifold of constant curvature $c$. Let $M^{n}$ be an $n$-dimensional Riemannian manifold immersed in $M^{n+p}$. Let $h$ be the second fundamental form of the immersion, and $\xi$ the mean curvature vector. Denote by $g$ the scalar product of $M^{n+p}$. If there exists a function $\lambda$ on $M^{n}$ such that

$$
g(h(X, Y), \xi)=\lg (X, Y)
$$


for any tangent vector $X, Y$ on $M^{n}$, then $M^{n}$ is called a pseudo-umbilical submanifold of $M^{n+p}$. It is clear that $\lambda \geq 0$. If the mean curvature $\xi=0$ identically, then $M^{n}$ is called a minimal submanifold of $M^{n+p}$.

Every minimal submanifold of $M^{n+p}$ is itself a pseudo umbilical submanifold of $M^{n+p}$. Cao [6] extended Bai's well-known theorem to the case in which $M^{n+p}$ is pseudoumbilical. The aim of the present work is to obtain this theorem for compact pseudo-umbilical submanifold of a Hessian manifold and also give some results and examples of it.

Theorem A. Let $M^{n}$ be an $n$-dimensional compact pseudo-umbilical submanifold of $(n+p)$ dimensional Hessian manifold of constant Hessian sectional curvature c. Then

$$
\begin{aligned}
\int_{M^{n}}\left\{p \sum R_{i j k l}^{2}+2 p \sum R_{i j}^{2}-R^{2}-n(3 p+2 n-2) \frac{c}{4} R-n(3 p-2 n) H^{2} R\right. \\
\left.+2 n^{2}(1-n) p \frac{c}{4} H^{2}+2 n^{3}(1-n) \frac{c}{4} H^{2}+n^{3} p H^{4}-n^{4} H^{4}\right\} * 1 \\
\geq n^{2}(n-1)(n-p-1) \frac{c^{2}}{16} \operatorname{Vol} M^{n},
\end{aligned}
$$

where $\sum R_{i j k l}^{2}$ is the square length of the Riemannian curvature tensor, $\sum R_{i j}^{2}$ is the square length of the Ricci curvature tensor, $R$ is the scalar curvature, and $H$ is the mean curvature of $M^{n}$.

We will use the same notation and terminologies as in [2] unless otherwise stated.

Let $M^{n+p}$ be a Hessian manifold with Hessian structure $(D, g)$. We express various geometric concepts for the Hessian structure $(D, g)$ in terms of affine coordinate system $\left\{x^{1}, \ldots, x^{n+p}\right\}$ with respect to $D$, that is, $D d x^{i}=0$.

(i) The Hessian metric,

$$
g_{i j}=\frac{\partial^{2} u}{\partial x^{i} \partial x^{j}}
$$

(ii) Let $\gamma$ be a tensor field of type $(1,2)$ defined by

$$
\gamma(X, Y)=\nabla_{X} Y-D_{X} Y
$$

where $\nabla$ is the Riemannian connection for $g$. Then we have

$$
\begin{gathered}
\gamma_{j k}^{i}=\Gamma_{j k}^{i}=\frac{1}{2} g^{i r} \frac{\partial g_{r j}}{\partial x^{k}} \\
\gamma_{i j k}=\frac{1}{2} \frac{\partial g_{i j}}{\partial x^{k}}=\frac{1}{2} \frac{\partial^{3} u}{\partial x^{i} \partial x^{j} \partial x^{k}} \\
\gamma_{i j k}=\gamma_{j i k}=\gamma_{k j i}
\end{gathered}
$$

where $\Gamma_{j k}^{i}$ are the Christoffel's symbols of $\nabla$. 
(iii) Define a tensor field $S$ of type $(1,3)$ by

$$
S=D_{\gamma}
$$

and call it the Hessian curvature tensor for $(D, g)$. Then we have

$$
\begin{gathered}
S_{j k l}^{i}=\frac{\partial \gamma_{j l}^{i}}{\partial x^{k}} \\
S_{i j k l}=\frac{1}{2} \frac{\partial^{4} u}{\partial x^{i} \partial x^{j} \partial x^{k} \partial x^{l}}-\frac{1}{2} g^{r s} \frac{\partial^{3} u}{\partial x^{i} \partial x^{k} \partial x^{r}}-\frac{\partial^{3} u}{\partial x^{j} \partial x^{l} \partial x^{s}} \\
S_{i j k l}=S_{i l k j}=S_{k j i l}=S_{j i l k}=S_{k l i j} .
\end{gathered}
$$

(iv) The Riemannian curvature tensor for $\nabla$,

$$
\begin{gathered}
R_{j k l}^{i}=\gamma_{r k}^{i} \gamma_{j l}^{r}-\gamma_{r l}^{i} \gamma_{j k}^{r} \\
R_{i j k l}=\frac{1}{2}\left(S_{j i k l}-S_{i j k l}\right)
\end{gathered}
$$

(see [2]).

Definition 1.1. Let $S_{j l}^{i k}$ be a Hessian curvature tensor on a Hessian manifolds $(M, D, g)$. We define an endomorphism $\varsigma$ of the space of contravariant symmetric tensor fields of degree 2 by

$$
\xi(\xi)^{i k}=S_{j l}^{i k} \xi^{j l}
$$

Then $\xi$ is a symmetric operator, [2].

Definition 1.2. For a nonzero contravariant symmetric tensor $\xi_{x}$ of degree 2 at $x$, we set

$$
h\left(\xi_{x}\right)=\frac{\left\langle\xi\left(\xi_{x}\right), \xi_{x}\right\rangle}{\left\langle\xi_{x}, \xi_{x}\right\rangle}
$$

and call it the Hessian sectional curvature in the direction $\xi_{x},[2]$.

Theorem 1.3. Let $\left(M^{n+p}, D, g\right)$ be a Hessian manifold of dimension $\geq 2$. If the Hessian sectional curvature $h\left(\xi_{x}\right)$ depends only on $x$, then $(M, D, g)$ is of constant Hessian sectional curvature. $(M, D, g)$ is of constant Hessian sectional curvature $c$ if and only if

$$
S_{i j k l}=\frac{c}{2}\left(g_{i j} g_{k l}+g_{i l} g_{k j}\right)
$$

(see [2]). 
Corollary 1.4. If a Hessian manifold $\left(M^{n+p}, D, g\right)$ is a space of constant Hessian sectional curvature $c$, then the Riemannian manifold $(M, g)$ is a space of constant sectional curvature $-c / 4,[2]$.

From now on, we shall construct, for each constant $c$, a Hessian manifold with constant Hessian sectional curvature $c$. We now recall the following result due to Shima and Yagi [7]. Let $\left(M^{n+p}, D, g\right)$ be a simply connected Hessian manifold. If $g$ is complete, then $\left(M^{n+p}, D, g\right)$ is isomorphic to $\left(\Omega, \widetilde{D}, \widetilde{D}^{2} \varphi\right)$, where $\Omega$ is a convex domain in $\mathbb{R}^{n+p}, \widetilde{D}$ is the canonical flat connection on $\mathbb{R}^{n+p}$, and $\varphi$ is a smooth convex function on $\Omega$.

(A) Case $c=0$

It is obvious that the Euclidean space $\left(\mathbb{R}^{n+p}, \tilde{D}, g=(1 / 2) \tilde{D}^{2}\left\{\sum_{A=1}^{n+p}\left(x^{A}\right)^{2}\right\}\right)$ is a simply connected Hessian manifold of constant Hessian sectional curvature 0 [1].

(B) Case $c>0$

Theorem 1.5. Let $\Omega$ be a domain in $\mathbb{R}^{n+p}$ given by

$$
x^{n+p}>\frac{c}{2} \sum_{A=1}^{n+p-1}\left(x^{A}\right)^{2}
$$

where $c$ is a positive constant, and let $\varphi$ be a smooth function on $\Omega$ defined by

$$
\varphi=-\frac{1}{c} \log \left\{x^{n+p}-\frac{c}{2} \sum_{A=1}^{n+p-1}\left(x^{A}\right)^{2}\right\} .
$$

Then $\left(\Omega, \widetilde{D}, g=\widetilde{D}^{2} \varphi\right)$ is a simply connected Hessian manifold of positive constant Hessian sectional curvature c. As Riemannian manifold $(\Omega, g)$ is isometric to the hyperbolic space $(H(-c / 4), g)$ of constant sectional curvature $-c / 4$;

$$
\begin{gathered}
H=\left\{\left(\xi^{1}, \ldots, \xi^{n}, \xi^{n+p}\right) \in \mathbb{R}^{n+p} \mid \xi^{n+p}>0\right\}, \\
g=\frac{1}{\left(\xi^{n+p}\right)^{2}}\left\{\sum_{A=1}^{n+p-1}\left(d \xi^{A}\right)^{2}+\frac{4}{c}\left(d \xi^{n+p}\right)^{2}\right\} .
\end{gathered}
$$

(C) Case $c<0$

Theorem 1.6. Let $\varphi$ be a smooth function on $\mathbb{R}^{n+p}$ defined by

$$
\varphi=-\frac{1}{c} \log \left(\sum_{A=1}^{n+p} e^{-c x^{A}}+1\right),
$$

where $c$ is a negative constant. Then $\left(\mathbb{R}^{n+p}, \widetilde{D}, g=\widetilde{D}^{2} \varphi\right)$ is a simply connected Hessian manifold of negative constant Hessian sectional curvature $c$. The Riemannian manifold $\left(\mathbb{R}^{n+p}, g\right)$ is isometric to a domain of the sphere $\sum_{i=1}^{n+p+1} \xi_{A}^{2}=-4 / c$ defined by $\xi_{A}>0$ for all $A$ [1].

For the proof of the theorems we refer to [1]. 


\section{Local Formulas}

We choose a local field of orthonormal frames $e_{1}, \ldots, e_{n+p}$ in $M^{n+p}$ such that restricted to $M^{n}, e_{1}, \ldots, e_{n}$ are tangent to $M^{n}$. Let $w_{1}, \ldots, w_{n+p}$ be its dual frame field. Then the structure equations of $M^{n+p}$ are given by

$$
\begin{gathered}
d w_{A}=-\sum w_{A B} \wedge w_{B}, \quad w_{A B}+w_{B A}=0 \\
d w_{A B}=-\sum w_{A C} \wedge w_{C B}+\frac{1}{2} \sum \bar{R}_{A B C D} w_{C} \wedge w_{D} .
\end{gathered}
$$

We restrict these forms to $M^{n}$, then we have

$$
\begin{gathered}
w_{\alpha}=0, \quad w_{\alpha i}=\sum h_{i j}^{\alpha} w_{j}, \quad h_{i j}^{\alpha}=h_{j i}^{\alpha} \\
d w_{i j}=-\sum w_{i k} \wedge w_{k j}+\frac{1}{2} \sum R_{i j k l} w_{k} \wedge w_{l}, \\
R_{i j k l}=-\frac{c}{4}\left(\delta_{i k} \delta_{j l}-\delta_{i l} \delta_{j k}\right)+\sum\left(h_{i k}^{\alpha} h_{j l}^{\alpha}-h_{i l}^{\alpha} h_{j k}^{\alpha}\right),
\end{gathered}
$$

where $R_{i j k l}$ are the components of the curvature tensor of $M^{n}$.

$$
\begin{gathered}
d w_{\alpha \beta}=-\sum w_{\alpha \gamma} \wedge w_{\gamma \beta}+\frac{1}{2} \sum R_{\alpha \beta k l} w_{k} \wedge w_{l,} \\
R_{\alpha \beta k l}=\sum\left(h_{i k}^{\alpha} h_{i l}^{\beta}-h_{i k}^{\beta} j h_{i l}^{\alpha}\right) .
\end{gathered}
$$

We call

$$
h=\sum h_{i j}^{\alpha} w_{i} w_{j} e_{\alpha}
$$

the second fundamental form of the immersed manifold $M^{n}$. Denote by

$$
S=\sum\left(h_{i j}^{\alpha}\right)^{2}
$$

the square length of $h, \xi=(1 / n) \sum \operatorname{tr} H_{\alpha} e_{\alpha}$ the mean curvature vector and $H=$ $(1 / n) \sqrt{\sum\left(\operatorname{tr} H_{\alpha}\right)^{2}}$ the mean curvature of $M^{n}$, respectively. Here tr is the trace of the matrix $H_{\alpha}=\left(h_{i j}^{\alpha}\right)$. Now let $e_{n+1}$ be parallel to $\xi$. Then we have

$$
\operatorname{tr} H_{n+1}=n H, \quad \operatorname{tr} H_{\alpha}=0, \quad \alpha \neq n+1 .
$$


Let $h_{i j k}^{\alpha}$ and $h_{i j k l}^{\alpha}$ denote the covariant derivative and the second covariant derivative of $h_{i j}^{\alpha}$, respectively, defined by

$$
\begin{gathered}
\sum h_{i j k}^{\alpha} w_{k}=d h_{i j}^{\alpha}-\sum h_{i k}^{\alpha} w_{k j}-\sum h_{j k}^{\alpha} w_{k i}-\sum h_{i j}^{\beta} w_{\beta \alpha} \\
\sum h_{i j k l}^{\alpha} w_{l}=d h_{i j k}^{\alpha}-\sum h_{i j l}^{\alpha} w_{l k}-\sum h_{i l k}^{\alpha} w_{l j}-\sum h_{l j k}^{\alpha} w_{l i} \sum h_{i j k}^{\beta} w_{\beta \alpha}
\end{gathered}
$$

Then we have

$$
\begin{gathered}
h_{i j k}^{\alpha}-h_{i k j}^{\alpha}=0, \\
h_{i j k l}^{\alpha}-h_{i j l k}^{\alpha}=\sum h_{i m}^{\alpha} R_{m j k l}+\sum h_{m j}^{\alpha} R_{m i k l}-\sum h_{i j}^{\beta} R_{\alpha \beta k l} .
\end{gathered}
$$

The Laplacian $\Delta h_{i j}^{\alpha}$ of $h_{i j}^{\alpha}$ is defined by $\Delta h_{i j}^{\alpha}=\sum h_{i j k k}^{\alpha}$. By a direct calculation we have

$$
\begin{aligned}
\frac{1}{2} \Delta S= & \sum\left(h_{i j k}^{\alpha}\right)^{2}+\sum h_{i j}^{\alpha} \Delta h_{i j}^{\alpha} \\
= & \sum\left(h_{i j k}^{\alpha}\right)^{2}+\sum h_{i j}^{\alpha} h_{k k i j}^{\alpha}+\sum h_{i j}^{\alpha} h_{m k}^{\alpha} R_{m i j k} \\
& +\sum h_{i j}^{\alpha} h_{m i}^{\alpha} R_{m k j k}-\sum h_{i j}^{\alpha} h_{k i}^{\beta} R_{\alpha \beta j k} .
\end{aligned}
$$

\section{Proof of Theorem A}

From $(*)$ and (2.6), we have

$$
g\left(h\left(e_{i}, e_{j}\right), H_{e_{n+1}}\right)=H^{2} \delta_{i j}
$$

therefore,

$$
\begin{gathered}
\sum h_{i j}^{\alpha} h_{k k i j}^{\alpha}=n H \Delta H, \\
\sum\left(h_{i j k}^{\alpha}\right)^{2} \geq \sum\left(h_{i i k}^{n+1}\right)^{2}=n \sum\left(\Delta_{i} H\right)^{2}=n|\nabla H|^{2} .
\end{gathered}
$$

It is obvious that

$$
\frac{1}{2} \Delta H^{2}=H \Delta H+|\nabla H|^{2}
$$

and, therefore,

$$
\sum\left(h_{i j k}^{\alpha}\right)^{2}+\sum h_{i j}^{\alpha} h_{k k i j}^{\alpha} \geq n|\nabla H|^{2}+n H \Delta H=\frac{1}{2} n \Delta H^{2} .
$$


On the other hand, from (2.2)

$$
\begin{aligned}
\sum h_{i j}^{\alpha} h_{m k}^{\alpha} R_{m i j k} & =\frac{1}{2}\left(h_{i j}^{\alpha} h_{m k}^{\alpha}-h_{m j}^{\alpha} h_{i k}^{\alpha}\right) R_{m i j k} \\
& =\frac{1}{2} \sum\left\{R_{i m j k}+\frac{c}{4}\left(\delta_{i j} \delta_{m k}-\delta_{m j} \delta_{i k}\right)\right\} R_{m i j k} \\
& =-\frac{1}{2} \sum R_{m i j k}^{2}-\frac{c}{4} R \\
\sum h_{i j}^{\alpha} h_{m i}^{\alpha} R_{m k j k} & =\sum\left\{(1-n) \frac{c}{4} \delta_{m j}+n H^{2} \delta_{m j}-R_{m j}\right\} R_{m j} \\
& =-\sum R_{m j}^{2}+(1-n) \frac{c}{4} R+n H^{2} R .
\end{aligned}
$$

From (2.3), we have

$$
\sum h_{i j}^{\alpha} h_{k i}^{\beta} R_{\alpha \beta j k}=\sum h_{i j}^{\alpha} h_{l j}^{\alpha} h_{k i}^{\beta} h_{l k}^{\beta}-\sum h_{i j}^{\alpha} h_{l k}^{\alpha} h_{i k}^{\beta} h_{l j}^{\beta}
$$

while

$$
\begin{aligned}
\sum h_{i j}^{\alpha} h_{l j}^{\alpha} h_{k i}^{\beta} h_{l k}^{\beta} & =\sum\left\{(1-n) \frac{c}{4} \delta_{i l}+n H^{2} \delta_{i l}-R_{i l}\right\}^{2} \\
& =n(1-n)^{2} \frac{c^{2}}{16}-2 n^{2}(1-n) \frac{c}{4} H^{2}-2(1-n) \frac{c}{4} R-2 n H^{2} R+n^{3} H^{4}+R_{i l}^{2} .
\end{aligned}
$$

Let

$$
S_{\alpha}=\sum_{i, j}\left(h_{i j}^{\alpha}\right)^{2}
$$

then we have

$$
S=\sum_{\alpha} S_{\alpha}
$$

Since

$$
\begin{gathered}
S^{2}=\left(\sum_{\alpha} S_{\alpha}\right)^{2}=\sum_{\alpha} S_{\alpha}^{2}+2 \sum_{\alpha<\beta} S_{\alpha} S_{\beta}, \\
\sum_{\alpha<\beta}\left(S_{\alpha}-S_{\beta}\right)^{2}=(p-1) \sum_{\alpha} S_{\alpha}^{2}-2 \sum_{\alpha<\beta} S_{\alpha} S_{\beta} \geqslant 0
\end{gathered}
$$

it follows that

$$
(p-1) \sum_{\alpha} S_{\alpha}^{2} \geqslant 2 \sum_{\alpha<\beta} S_{\alpha} S_{\beta}=S^{2}-\sum_{\alpha} S_{\alpha}^{2}
$$


that is

$$
\sum_{\alpha} S_{\alpha}^{2} \geq \frac{1}{p} S^{2}
$$

Since

$$
\begin{aligned}
\sum_{i, j, l, k}\left(h_{i j}^{\alpha} h_{l k}^{\alpha}\right)^{2} & =\sum_{i, j, l, k}\left(\sum_{\beta} h_{i k}^{\beta} h_{l j}^{\beta}\right)^{2} \\
& =\sum_{\alpha, \beta} h_{i j}^{\alpha} h_{i j}^{\beta} h_{l k}^{\alpha} h_{l k}^{\beta} \\
& =\sum_{\alpha, \beta}\left(\sum_{i, j} h_{i j}^{\alpha} h_{i j}^{\beta}\right)^{2} \geq \sum_{\alpha}\left(\sum_{i, j} h_{i j}^{\alpha} h_{i j}^{\alpha}\right)^{2}=\sum_{\alpha} S_{\alpha}^{2}
\end{aligned}
$$

we have

$$
\begin{aligned}
\sum_{\alpha, \beta} h_{i j}^{\alpha} h_{l k}^{\alpha} h_{i k}^{\beta} h_{l j}^{\beta} & \geq \sum_{\alpha, \beta} h_{i j}^{\alpha} h_{l k}^{\alpha} h_{i k}^{\beta} h_{l j}^{\beta}-\frac{1}{2} \sum_{i, j, l, k}\left(\sum_{\alpha} h_{i j}^{\alpha} h_{l k}^{\alpha}\right)^{2}-\frac{1}{2} \sum_{i, j, l, k}\left(\sum_{\beta} h_{i k}^{\beta} h_{l j}^{\beta}\right)^{2}+\sum_{\alpha} S_{\alpha}^{2} \\
& =-\frac{1}{2} \sum\left\{\sum_{\alpha}\left(h_{i j}^{\alpha} h_{l k}^{\alpha}-h_{i k}^{\alpha} h_{l j}^{\alpha}\right)\right\}^{2}+\sum_{\alpha} S_{\alpha}^{2} \\
& =-\frac{1}{2} \sum\left\{R_{i l j k}+\frac{c}{4}\left(\delta_{i j} \delta_{l k}-\delta_{i k} \delta_{l j}\right)\right\}^{2}+\sum_{\alpha} S_{\alpha}^{2} \\
& \geq-\frac{1}{2} \sum R_{i l j k}^{2}-2 \frac{c}{4} R-n(1-n) \frac{c^{2}}{16}+\frac{1}{p} S^{2} .
\end{aligned}
$$

From (2.9), (3.4)-(3.14), we have

$$
\begin{aligned}
\frac{1}{2} \Delta S \geq & \frac{1}{2} n \Delta H^{2}-\sum R_{i j k l}^{2}-2 \sum R_{i j}^{2}-\frac{3}{4} c n R+3 n H^{2} R \\
& -n^{2}(1-n) \frac{c^{2}}{16}-2 n^{2}(1-n) \frac{c}{4} H^{2}-n^{3} H^{4}+\frac{1}{p} S^{2} .
\end{aligned}
$$

Since $M^{n}$ is compact and

$$
S=n(1-n) \frac{C}{4}+n^{2} H^{2}-R
$$


we have

$$
\begin{aligned}
& \int_{M^{n}}\left\{p \sum R_{i j k l}^{2}+2 p \sum R_{i j}^{2}+\frac{3}{4} c n p R-3 n p H^{2} R+n^{2}(1-n) \frac{c^{2}}{16} p\right. \\
& \left.+2 n^{2}(1-n) \frac{c}{4} H^{2} p+n^{3} H^{4} p-S^{2}\right\} * 1 \\
& S^{2}=n^{2}(1-n)^{2} \frac{c^{2}}{16}+n^{4} H^{4}+R^{2}+2 n^{3}(1-n) H^{2} \frac{c}{4}-2 n^{2} H^{2} R-2 n(1-n) \frac{c}{4} R \\
& \int_{M^{n}}\left\{p \sum R_{i j k l}^{2}+2 p \sum R_{i j}^{2}+\frac{3}{4} n c R p-3 n H^{2} R p+n^{2}(1-n) \frac{c^{2}}{16} p\right. \\
& +2 n^{2}(1-n) \frac{c}{4} H^{2} p+n^{3} H^{4} p-n^{2}(1-n)^{2} \frac{c^{2}}{16}-n^{4} H^{4}-R^{2} \\
& \left.-2 n^{3}(1-n) H^{2} \frac{c^{2}}{16}+2 n^{2} H^{2} R+2 n(1-n) \frac{c}{4} R\right\} * 1
\end{aligned}
$$

and we have

$$
\begin{aligned}
\int_{M^{n}}\{ & p \sum R_{i j k l}^{2}+2 p \sum R_{i j}^{2}-R^{2}+n(3 p-2 n+2) \frac{c}{4} R-n(3 p-2 n) H^{2} R \\
& \left.+2 n^{2}(1-n) p \frac{c}{4} H^{2}+2 n^{3}(1-n) \frac{c}{4} H^{2}+n^{3} p H^{4}-n^{4} H^{4}\right\} * 1 \\
\geq & n^{2}(1-n)(n-p-1) \frac{c^{2}}{16} \operatorname{Vol}\left(M^{n}\right) .
\end{aligned}
$$

Corollary 3.1. Let $M^{n}$ be an $n$-dimensional compact pseudo-umbilical submanifold of $\left(\mathbb{R}^{n+p}, \tilde{D}, g=\right.$ $\left.(1 / 2) \tilde{D}^{2}\left\{\left(x^{A}\right)^{2}\right\}\right)$. Then

$$
\int_{M^{n}}\left\{p \sum R_{i j k l}^{2}+2 p \sum R_{i j}^{2}-R^{2}-n(3 p-2 n) H^{2} R+n^{3} p H^{4}-n^{4} H^{4}\right\} * 1 \geq 0 .
$$

Proof. The Euclidean space $\left(\mathbb{R}^{n+p}, \widetilde{D}, g=(1 / 2) \widetilde{D}^{2}\left\{\sum_{A=1}^{n+p}\left(x^{A}\right)^{2}\right\}\right)$ is a simply connected Hessian manifold of constant Hessian sectional curvature 0. Taking into account of Theorem A, we conclude the corollary.

Corollary 3.2. Let $\Omega$ be a domain in $\mathbb{R}^{n+p}$ given by

$$
x^{n+p}>\frac{c}{2} \sum_{A=1}^{n+p-1}\left(x^{A}\right)^{2}
$$


where $c$ is a positive constant, and let $\varphi$ be a smooth function on $\Omega$ defined by

$$
\varphi=-\frac{1}{c} \log \left\{x^{n+p}-\frac{c}{2} \sum_{A=1}^{n+p-1}\left(x^{A}\right)^{2}\right\}
$$

Let $M^{n}$ be an $n$-dimensional compact pseudo-umbilical submanifold of $\left(\Omega, \widetilde{D}, g=\widetilde{D}^{2} \varphi\right)$. Then Theorem A holds.

Proof. It is obvious that $\left(\Omega, \tilde{D}, g=\widetilde{D}^{2} \varphi\right)$ is a simply connected Hessian manifold of positive constant Hessian sectional curvature $c$. As Riemannian manifold $(\Omega, g)$ is isometric to the hyperbolic space $(H(-c / 4), g)$ of constant sectional curvature $-c / 4$;

$$
\begin{gathered}
H=\left\{\left(\xi^{1}, \ldots, \xi^{n}, \xi^{n+p}\right) \in \mathbb{R}^{n+p} \mid \xi^{n+p}>0\right\}, \\
g=\frac{1}{\left(\xi^{n+p}\right)^{2}}\left\{\sum_{A=1}^{n+p-1}\left(d \xi^{A}\right)^{2}+\frac{4}{c}\left(d \xi^{n+p}\right)^{2}\right\} .
\end{gathered}
$$

As a consequence of Theorem A, we conclude the proof.

On the other hand let us define $\varphi$ as a smooth function on $\mathbb{R}^{n+p}$ as follows

$$
\varphi=-\frac{1}{c} \log \left(\sum_{A=1}^{n+p} e^{-c x^{A}}+1\right)
$$

where $c$ is a negative constant. Then $\left(\mathbb{R}^{n+p}, \widetilde{D}, g=\widetilde{D}^{2} \varphi\right)$ is a simply connected Hessian manifold of negative constant Hessian sectional curvature $c$. The Riemannian manifold $\left(\mathbb{R}^{n+p}, g\right)$ is isometric to a domain of the sphere $\sum_{i=1}^{n+p+1} \xi_{A}^{2}=-4 / c$ defined by $\xi_{A}>0$ for all $A$. Hence we acquire the following.

Corollary 3.3. Let $\varphi$ be a smooth function on $\mathbb{R}^{n+p}$ defined by

$$
\varphi=-\frac{1}{c} \log \left(\sum_{A=1}^{n+p} e^{-c x^{A}}+1\right),
$$

where $c$ is a negative constant and $M^{n}$ be an $n$-dimensional compact pseudo-umbilical submanifold of $\left(\mathbb{R}^{n+p}, \tilde{D}, g=\widetilde{D}^{2} \varphi\right)$. Then

$$
\begin{aligned}
\int_{M^{n}} & \left\{p \sum R_{i j k l}^{2}+2 p \sum R_{i j}^{2}-R^{2}+n(3 p+2 n-2) \frac{c}{4} R-n(3 p-2 n) H^{2} R\right. \\
& \left.-2 n^{2}(1-n) p \frac{c}{4} H^{2}-2 n^{3}(1-n) \frac{c}{4} H^{2}+n^{3} p H^{4}-n^{4} H^{4}\right\} * 1 \\
\geq & n^{2}(n-1)(n-p-1) \frac{c^{2}}{16} \operatorname{Vol} M^{n} .
\end{aligned}
$$




\subsection{Applications in 3-Dimensional Spaces}

Here we give some examples of the results indicated above.

Example 3.4. Let $M^{2}$ be a 2-dimensional compact pseudo-umbilical surface of $\left(\mathbb{R}^{3}, \tilde{D}, g=\right.$ $\left.(1 / 2) \tilde{D}^{2}\left\{\left(x^{A}\right)^{2}\right\}\right)$. Then

$$
\int_{M^{2}} \sum R_{i j k l}^{2}+2 \sum R_{i j}^{2}-R^{2}-2 H^{2}\left(R+6 H^{4}\right)>0
$$

also note that if the Ricci curvature tensor of the surface is given by $R_{i j}=K g_{i j}$, we may also compute the integral $i$ terms of Gaussian curvature K.

Example 3.5. Let $\Omega$ be a domain in $\mathbb{R}^{3}$ given by

$$
x^{3}>\frac{c}{2}\left[\left(x^{1}\right)^{2}+\left(x^{2}\right)^{2}\right]
$$

where $c$ is a positive constant, and let $\varphi$ be a smooth function on $\Omega$ defined by

$$
\varphi=-\frac{1}{c} \log \left\{x^{3}-\frac{c}{2}\left[\left(x^{1}\right)^{2}+\left(x^{2}\right)^{2}\right]\right\} .
$$

Let $M^{2}$ be a 2-dimensional compact pseudo-umbilical surface of $\left(\Omega, \widetilde{D}, g=\widetilde{D}^{2} \varphi\right)$. Then

$$
\int_{M^{2}} \sum R_{i j k l}^{2}+2 \sum R_{i j}^{2}-R^{2}-\frac{5}{2} c R+2 H^{2}\left(R-3 c-4 H^{2}\right)>0 .
$$

Example 3.6. Let $\varphi$ be a smooth function on $\mathbb{R}^{3}$ defined by $c$

$$
\varphi=-\frac{1}{c} \log \left(\sum_{A=1}^{3} e^{-c x^{A}}+1\right)
$$

where $c$ is a negative constant and $M^{2}$ be a 2-dimensional compact pseudo-umbilical surface of $\left(\mathbb{R}^{3}, \tilde{D}, g=\widetilde{D}^{2} \varphi\right)$. Then

$$
\int_{M^{2}} \sum R_{i j k l}^{2}+2 \sum R_{i j}^{2}-R^{2}+\frac{3}{2} c R+2 H^{2}\left(R+3 c-4 H^{2}\right)>0
$$

and $\left(\mathbb{R}^{3}, g\right)$ is isometric to a domain of the sphere $\mathbb{S}^{3}(\sqrt{-4 / c})$. 


\section{References}

[1] H. Shima, The Geometry of Hessian Structures, World Scientific, Hackensack, NJ, USA, 2007.

[2] H. Shima, "Hessian manifolds of constant Hessian sectional curvature," Journal of the Mathematical Society of Japan, vol. 47, no. 4, pp. 735-753, 1995.

[3] M. Bektaş and M. Yıldırım, "Integral inequalities for submanifolds of Hessian manifolds with constant Hessian sectional curvature," Iranian Journal of Science and Technology. Transaction A, vol. 30, no. 2, pp. 235-239, 2006.

[4] M. Y. Yilmaz and M. Bektaş, "A survey on curvatures of Hessian manifolds," Chaos, Solitons and Fractals, vol. 38, no. 3, pp. 620-630, 2008.

[5] M. Bektaş, M. Yildirim, and M. Külahci, "On hypersurfaces of Hessian manifolds with constant Hessian sectional curvature," Journal of Mathematics and Statistics, vol. 1, no. 2, pp. 115-118, 2005.

[6] X.-F. Cao, "Pseudo-umbilical submanifolds of constant curvature Riemannian manifolds," Glasgow Mathematical Journal, vol. 43, no. 1, pp. 129-133, 2001.

[7] H. Shima and K. Yagi, "Geometry of Hessian manifolds," Differential Geometry and its Applications, vol. 7, no. 3, pp. 277-290, 1997. 


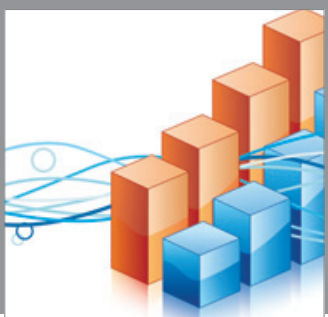

Advances in

Operations Research

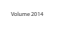

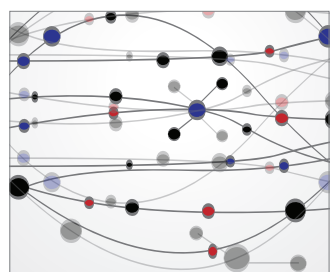

\section{The Scientific} World Journal
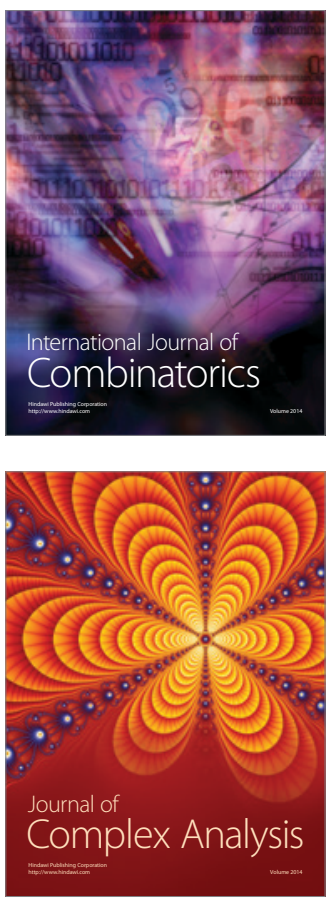

International Journal of

Mathematics and

Mathematical

Sciences
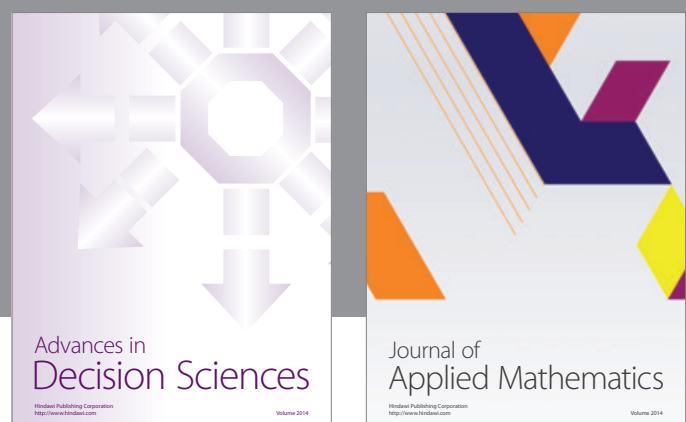

Journal of

Applied Mathematics
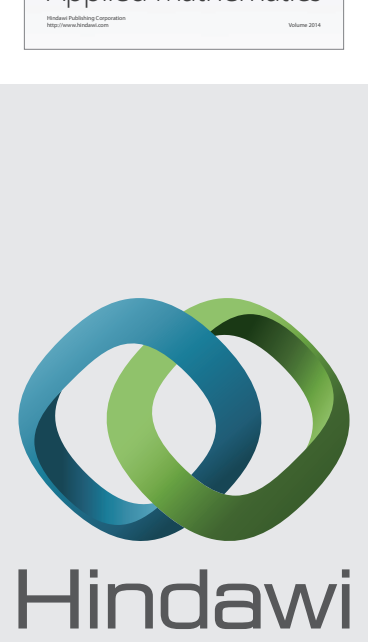

Submit your manuscripts at http://www.hindawi.com
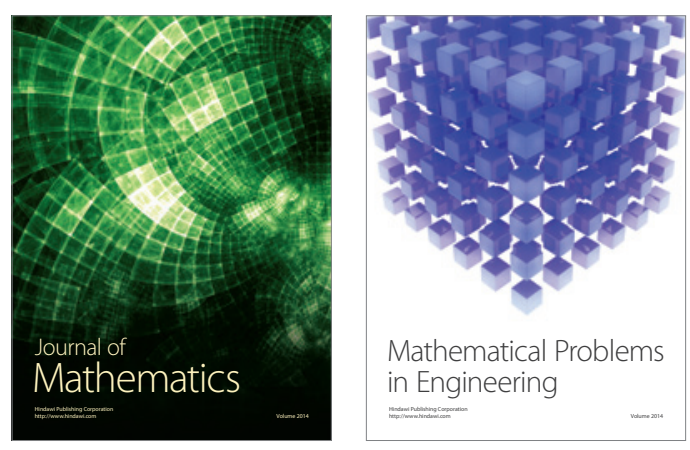

Mathematical Problems in Engineering
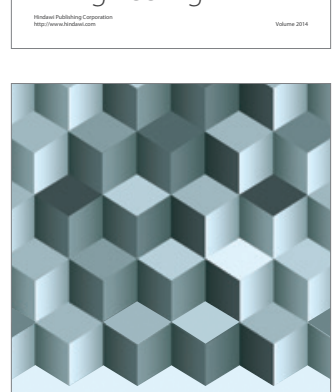

Journal of

Function Spaces
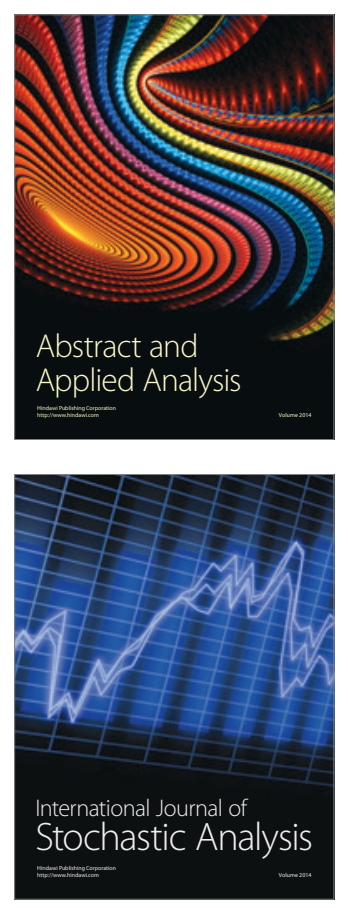

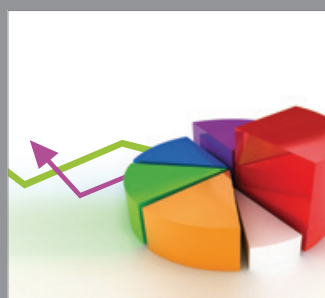

ournal of

Probability and Statistics

Promensencen
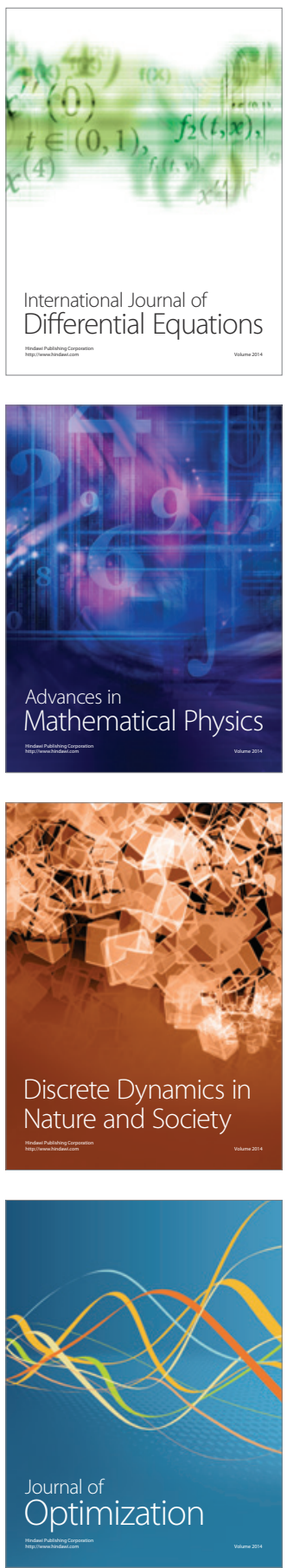